\title{
Primary Caregivers of Children Affected by Disorders of Sex Development : Mental Health and Caregiver Characteristics in the Context of Genital Ambiguity and Genitoplasty
}

\author{
David A. Fedele, ${ }^{1}$ Katherine Kirk, ${ }^{2}$ Cortney Wolfe-Christensen, ${ }^{3}$ Timothy M. Phillips, ${ }^{4}$ \\ Tom Mazur, ${ }^{5}$ Larry L. Mullins, ${ }^{1}$ Steven D. Chernausek, ${ }^{6}$ and Amy B. Wisniewski ${ }^{6}$ \\ ${ }^{1}$ Department of Psychology, Oklahoma State University, Stillwater, OK 74078, USA \\ ${ }^{2}$ University of Oklahoma College of Nursing, Oklahoma City, OK 73117, USA \\ ${ }^{3}$ Department of Pediatrics/Neurology, Children's Hospital of Michigan, Detroit, MI 48201, USA \\ ${ }^{4}$ Brady Urological Institute, Johns Hopkins Medical Institutions, Baltimore, MD 21236, USA \\ ${ }^{5}$ Departments of Psychiatry and Pediatrics, School of Medicine, State University of New York at Buffalo, \\ The Women and Children's Hospital of Buffalo, Buffalo, NY 14222, USA \\ ${ }^{6}$ Department of Pediatrics, University of Oklahoma Health Sciences Center, Oklahoma City, OK 73104, USA
}

Correspondence should be addressed to Amy B. Wisniewski, amy-wisniewski@ouhsc.edu

Received 9 March 2010; Accepted 6 April 2010

Academic Editor: Peter Allen Lee

Copyright ( 2010 David A. Fedele et al. This is an open access article distributed under the Creative Commons Attribution License, which permits unrestricted use, distribution, and reproduction in any medium, provided the original work is properly cited.

\begin{abstract}
Purpose. To determine the relationship between having a child with a DSD including ambiguous external genitalia, as well as the decision of whether or not to have early genitoplasty for that child, on the mental health and parenting characteristics of caregivers. Materials and Methods. Caregivers were recruited from centers that specialize in DSD medicine and completed the Beck Depression Inventory 2nd Edition (BDI-2), Beck Anxiety Index (BAI), Parent Protection Scale (PPS), Child Vulnerability Scale (CVS) and Parenting Stress Index/Short Form (PSI/SF). Results and Conclusions. Sixty-eight caregivers provided informed consent and completed the study. Among female caregivers whose children never received genitoplasty, greater parenting stress was reported $(F(1,40)=5.08, p=.03)$. For male caregivers, those whose children received genitoplasty within the first year of life reported more overprotective parenting and parenting stress than those whose children received genitoplasty later than 12 months of age $(F(1,13)=6.16, p=0.28) ; F(1,15)=6.70, p=.021)$, respectively).
\end{abstract}

\section{Introduction}

The term Disorders of Sex Development (DSD) refers to congenital conditions in which development of chromosomal, gonadal, and phenotypic sex is discordant [1]. Studies have demonstrated higher levels of psychological distress and poorer sexual quality of life in people with DSD compared to unaffected individuals [1-4], although the reasons for these differences are unclear. Limited research has been conducted on how having a child with a DSD affects caregivers, especially with regard to their mental health and parenting characteristics [5]. In the extant chronic medical illness literature, researchers have found that parents of children with a chronic illness are at risk for (1) exhibiting levels of overprotection, (2) perceiving their child as more vulnerable than others, and (3) reporting significant levels of parenting stress [6-9]. Importantly, these parental characteristics are predictive of children's emotional, behavioral, and social adjustment $[10,11]$.

Rearing a child in whom genital ambiguity persists versus opting for genitoplasty (clitoroplasty, vaginoplasty, or hypospadias repair) before a child can assent to such procedures is a challenge for caregivers of children with DSD [12]. The 2006 consensus statement on DSD notes "It is generally felt that surgery that is performed for cosmetic reasons in the first year of life relieves parental distress and 
improves attachment between the child and parent etc." [1]. Indirect evidence for this is provided by the fact that some parents choose early genitoplasty for their children despite the acknowledged risk to sexual function resulting from such procedures $[13,14]$. The aim of the present study was to determine the related impact of having a child born with a DSD including ambiguous external genitalia, as well as the decision to have early genitoplasty for that child, on the mental health status and parenting characteristics of primary caregivers.

\section{Materials and Methods}

2.1. Participants and Procedure. Primary caregivers of children with DSD were invited to participate if their child was diagnosed with a DSD for at least 6 months prior to recruitment. The 6-month cut-off was chosen to ensure that caregivers of children newly diagnosed with DSD were not included as they are expected to exhibit elevated levels of depressive symptoms, anxiety and overprotective parenting. In families that included two caregivers, both were invited to participate. Families served by DSD specialists at the University of Oklahoma Children's Hospital, Johns Hopkins Hospital, and the Women's and Children's Hospital of Buffalo were invited to participate. Additionally, other parents could participate if they initiated contact with the senior author. Inclusion criteria included being 18 years of age or older, able to provide informed consent, and able to read English at an 8th grade reading level.

After obtaining informed consent, primary caregivers were mailed a questionnaire packet including a stamped return envelope. Following receipt of the completed questionnaire packet, a gift card worth $\$ 25$ was mailed to each participant. A total of 72 primary caregivers of 47 children were invited to participate (Tables 1 and 2). Four caregivers declined and 68 (40 mothers, 23 fathers, 1 grandmother, 1 grandfather, and 3 foster parents) provided informed consent and completed the study. All participants endorsed being primary caregivers for the child of interest and were recruited from a larger study examining caregiver adjustment to children with DSD.

2.2. Measures. For all measures employed, the following conventions were used to categorize internal and test-retest reliability estimates: $>.70$ is adequate, $>.80$ is good, and $>.90$ is excellent [15].

Demographics Questionnaire. Caregivers completed a questionnaire that assessed their age and race, child age, number of children and caregivers in the home, marital status, and household income.

Beck Anxiety Index (BAI). Caregiver levels of anxiety were measured with the BAI, a 21-item self-report measure developed for 17-80 year olds that uses a 4-point response scale ranging from "not at all" to "severely." Higher scores indicate greater levels of parental anxiety. Total scores can be categorized as minimal (0-7), mild (8-15), moderate (1625), and severe (26-63) [16]. The BAI has good psychometric
TABLE 1: General characteristics of caregiver participants $(n=68)$.

\begin{tabular}{lc}
\hline Variable & $M=35.4(19-55)$ \\
\hline Age (yr) & 71.6 \\
Married (\%) & \\
Ethnicity (\%) & 73.1 \\
$\quad$ Caucasian & 9.0 \\
$\quad$ African American & 7.5 \\
American Indian & 4.5 \\
Asian American & 3.0 \\
Hispanic & 3.0 \\
Other & \\
Annual household income (\%) & 9.1 \\
$<$ \$10,000 & 10.6 \\
\$10,000-19,999 & 6.1 \\
\$20,000-29,999 & 7.6 \\
$\$ 30,000-39,999$ & 21.2 \\
\$40,000-49,999 & 4.5 \\
\$50,000-59,999 & 40.9 \\
\$60,000+
\end{tabular}

TABLE 2: General characteristics of participants' children affected by DSD including genital ambiguity $(n=47)$.

\begin{tabular}{|c|c|}
\hline Variable & \\
\hline Age (yr) & $M=5.88(0.5-17.8)$ \\
\hline \multicolumn{2}{|l|}{ Diagnosis } \\
\hline 46,XY DSD* & $n=14$ \\
\hline $46, \mathrm{XX} \mathrm{DSD}^{\#}$ & $n=33$ \\
\hline Duration of diagnosis (yr) & $M=6.2(0.5-17.8)$ \\
\hline \multicolumn{2}{|l|}{ Received genitoplasty } \\
\hline$\leq 12$ mos age $(\%)$ & $n=21(63.3)$ \\
\hline$>12$ mos age $(\%)^{\dagger}$ & $n=12(36.4)$ \\
\hline \multicolumn{2}{|l|}{ Received genitoplasty } \\
\hline XX DSD & $n=25$ \\
\hline XY DSD & $n=8$ \\
\hline Reared male & $n=14$ \\
\hline Genital phenotype at birth & $M$ Quigley rating $=3.4$ \\
\hline Reared female & $n=33$ \\
\hline Genital phenotype at birth & mean Prader rating $=3.1$ \\
\hline
\end{tabular}

* Children with 46,XY DSD were affected by partial androgen insensitivity syndrome, partial gonadal dysgenesis, or an unknown cause. ${ }^{\#}$ Children with 46,XX DSD were affected by congenital adrenal hyperplasia due to 21-hydroxylase deficiency or transposition of Sry onto the X chromosome. ${ }^{\dagger}$ For children who received genitoplasty later than 12 mos of age, mean age for surgery was $22.8 \mathrm{mos}(\mathrm{SD}=5.9 \mathrm{mos})$.

properties including high internal reliability $(\alpha=0.92)$ and good test-retest reliability $(r=0.75)$ after one week $[16,17]$. Cronbach's alpha for the current sample was 0.86 meaning that the internal reliability for the measure was good.

Beck Depression Inventory-2nd Edition (BDI-2). Caregiver levels of depression were measured with the BDI-2, a 21-item self-report measure developed for 13-80 year olds that uses a 4-point response scale ranging from "not at all" to "severely." 
Higher BDI-2 scores indicate greater levels of depressive symptomology. Total scores can be categorized as minimal $(0-13)$, mild (14-19), moderate (20-28), and severe (2963) [18]. The BDI-2 possesses excellent internal reliability $(\alpha$ $=0.90-0.92)$ and test-retest reliability $(r=0.93)$ after one week $[18,19]$. Cronbach's alpha for the current sample was 0.92 meaning that the internal reliability for the measure was excellent.

Parent Protection Scale (PPS). Caregiver overprotection was measured with the PPS, a 25-item self-report that assesses dimensions of protective parenting of children ages 2-18 years. Caregivers use a 4-point scale ranging from "never" to "always" to answer each question. Higher scores indicate greater levels of overprotection of their child. A clinical cutoff of $1 \mathrm{SD}$ above the mean of the sample indicates clinically significant overprotection [20]. The PPS shows moderate to high internal reliability $(\alpha=0.73)$, high test-retest reliability $(r=0.86)$, and adequate internal consistency $(\alpha=0.74)$ [20]. Cronbach's alpha for the current sample was 0.76 meaning that the internal reliability for the measure was adequate.

Child Vulnerability Scale (CVS). Caregiver perception of child vulnerability was measured with the CVS, an 8-item self-report that uses a 4-point scale ranging from "definitely false" to "definitely true." Higher scores indicate greater perceived child vulnerability by caregivers. The CVS was originally validated on parents of children ageing $4-8$ years and includes a clinical cut-off score of 10 or greater to determine significant perceived vulnerability [21]. The CVS shows moderate to high internal reliability $(\alpha=0.74)$, high test-retest reliability $(r=0.84)$, and adequate internal consistency $(\alpha=0.78)$ [21]. Cronbach's alpha for the current sample was 0.76 meaning that the internal reliability for the measure was adequate.

Parenting Stress Index/Short Form (PSI/SF). Caregiver stress was measured with the PSI/SF, a 36-item self-report that uses a 5-point scale ranging from "strongly agree" to "strongly disagree." Higher PSI/SF scores indicate greater caregiver stress. The PSI/SF was developed for caregivers of children from 1 month to 12 years of age. A clinical cut-off score of 90 or higher indicates clinically significant caregiver stress [22]. The PSI/SF shows high internal consistency $(\alpha=0.92)$ and its validity has been established in parents of children with chronic medical conditions such as diabetes and asthma [22-24]. Cronbach's alpha for the current sample was 0.95 meaning that the internal reliability for the measure was excellent.

Statistical Analyses. Since male and female caregivers of the same child completed measures in many instances, separate analyses were conducted according to sex. The dataset was examined for missing values. If $5 \%$ or less of items were missing from any single measure, participant-specific mean values were inserted. If more than $5 \%$ of items were missing from any single measure, pairwise deletion was applied for that measure. In order to control for demographic (i.e., caregiver age, child age, annual income, number of children in the household and number of caregivers in the household) or illness characteristics (i.e., duration of illness) that may be related to the dependent variables of interest (i.e., BAI, BDI2, CVS, PPS, and PSI/SF total scores), bivariate correlations were conducted. Specifically, if a significant correlation was found between a demographic or illness variable and a dependent variable, that demographic or illness variable was used as a covariate in subsequent analyses (see Tables 3 and 4). Therefore, conducting these correlational analysis allowed us to control for any demographic or illness characteristics that were found to be significantly related to the dependent variables in order to provide a more stringent test of group differences. First, univariate analyses (ANOVA or ANCOVA) were conducted to determine whether caregivers of children who underwent genitoplasty differed from caregivers of children who did not have genitoplasty on all dependent measures, with applicable covariates entered for each comparison. To determine whether the child's age at the time of surgery (e.g., $\leq 1$ year old versus $>1$ year old) was related to any of the dependent variables, univariate analyses (ANOVA or ANCOVA) were conducted separately for male and female caregivers. The assumptions of homogeneity of variances and regression slopes were tenable for all analyses. All reported $\mathrm{P}$ values are two sided, statistical significance was considered at alpha $\leq 0.05$, and partial $\eta^{2}$ was used for an effect size estimate because it estimates the factor of interest while controlling for covariates. Statistical analyses were conducted with SPSS version 17.

\section{Results}

To determine whether male caregivers of children who had genitoplasty differed from male caregivers whose children did not, questionnaire responses were compared using univariate analyses while controlling for appropriate covariates (Table 5). Male caregivers did not differ on any of the dependent variables regardless of whether or not their child had ever received genitoplasty.

To determine if female caregivers of children who did, or did not, have genitoplasty differed on BAI, BDI-2, PPS, CVS, and PSI/SF total scores, univariate analyses controlling for appropriate covariates were conducted. Among female caregivers whose children did not receive genitoplasty, significantly higher levels of parenting stress were reported $(F(1,40)=5.08, P=.030$; Table 5$)$ as indicated by the PSI/SF.

To determine whether participants' mental health or parenting characteristics were affected by their child's age at the time of genitoplasty (i.e., $\leq 1$ year old versus $>1$ year old), univariate analyses controlling for applicable covariates were conducted for male and female caregivers. For males, those whose children had genitoplasty early had higher levels of overprotective parenting and parenting stress than those whose children received genitoplasty after 12 months of age $(F(1,13)=6.16, P=.028 ; F(1,15)=6.70, P=.021$, resp. $)$ as indicated by the PPS and PSI/SF, respectively. No relationship between the timing of surgery, mental health, and parenting characteristics was observed for female caregivers (Table 6). Neither gender of rearing of the child nor type of DSD was 
TABLE 3: Bivariate correlations to determine covariates for male and female guardians of children eligible for genitoplasty.

\begin{tabular}{|c|c|c|c|c|c|c|c|c|c|c|c|}
\hline Male Caregiver & 1 & 2 & 3 & 4 & 5 & 6 & 7 & 8 & 9 & 10 & 11 \\
\hline (1) Guardian Age & & $.51^{(* *)}$ & $.50^{(* *)}$ & .13 & $.41^{*}$ & $.51^{(* *)}$ & -.27 & -.18 & -.34 & -.27 & -.36 \\
\hline (2) Child Age & & & $.41^{*}$ & $.46^{*}$ & .35 & $1.00^{* *}$ & .29 & .18 & $-.83^{* *}$ & $-.40^{*}$ & -.09 \\
\hline (3) Annual Income & & & & .08 & $.59 * *$ & $.42 *$ & .22 & .29 & -.25 & .00 & -.13 \\
\hline (4) Number of Children in Home & & & & & .15 & $.45^{*}$ & -.17 & -.16 & $-.53^{* *}$ & $-.40^{*}$ & -.15 \\
\hline (5) Number of Caregivers in Home & & & & & & .36 & .15 & .18 & $-.41^{*}$ & .14 & .29 \\
\hline (6) Illness Duration & & & & & & & .28 & .18 & $-.82^{* *}$ & -.40 & -.08 \\
\hline (7) BAI Total & & & & & & & & $.82^{* *}$ & -.32 & .08 & $.43^{*}$ \\
\hline (8) BDI-2 Total & & & & & & & & & -.06 & .24 & $.44^{*}$ \\
\hline (9) PPS Total & & & & & & & & & & $48^{*}$ & -.03 \\
\hline (10) CVS Total & & & & & & & & & & & $.49^{*}$ \\
\hline \multicolumn{12}{|l|}{ (11) PSI/SF Total } \\
\hline Female Caregiver & 1 & 2 & 3 & 4 & 5 & 6 & 7 & 8 & 9 & 10 & 11 \\
\hline (1) Guardian Age & & $.53^{* *}$ & $.56^{* *}$ & .23 & .27 & $.55^{*}$ & .09 & -.03 & $-.38^{*}$ & .25 & -.24 \\
\hline (2) Child Age & & & .08 & $.40^{* *}$ & -.12 & $.88^{* *}$ & -.01 & -.04 & $-.60^{* *}$ & .12 & .00 \\
\hline (3) Annual Income & & & & .14 & $.57^{* *}$ & -.02 & .04 & -.02 & -.06 & .28 & -.19 \\
\hline (4) Number of Children in home & & & & & .09 & .30 & -.02 & -.20 & $-.34^{*}$ & .20 & -.21 \\
\hline (5) Number of Caregivers in home & & & & & & -.12 & .14 & -.28 & .10 & .07 & -.12 \\
\hline (6) Illness Duration & & & & & & & .08 & .04 & $-.70^{* *}$ & .14 & -.01 \\
\hline (7) BAI Total & & & & & & & & $.45^{* *}$ & .11 & .23 & $-.35^{*}$ \\
\hline (8) BDI-2 Total & & & & & & & & & .05 & .20 & $.68^{* *}$ \\
\hline (9) PPS Total & & & & & & & & & & -.10 & .02 \\
\hline (10) CVS Total & & & & & & & & & & & .03 \\
\hline (11) PSI/SF Total & & & & & & & & & & & \\
\hline
\end{tabular}

${ }^{*} P<.05$. ${ }^{* *} p<.01$. Significant demographic and illness correlations with dependent variables of interest were included as covariates in applicable analyses. Numerals with variable name correspond to numeral on horizontal heading.

related to BAI, BDI-2, PPS, CVS, or PSI/SF total scores for either male or female caregivers $(P>.05)$.

\section{Discussion}

The primary goal of the current study was to measure selfreported parenting characteristics, anxiety, and depressive symptoms in caregivers of children affected by DSD including ambiguous genitalia. Additionally, we investigated how the decision to proceed with genitoplasty early in a child's life related to caregivers' mental health and parenting characteristics. Female caregivers reported more parenting-related stress if their child's genitalia remained ambiguous (although the mean PSI for this group was not clinically significant). Interestingly, a recent study of children born with cleft lip revealed increased difficulties in mother-infant interactions when children underwent late (3-4 months), compared to early surgical intervention [25]. Possibly female caregivers are more stressed than males by a child's atypical physical appearance because females take primary responsibility for their child's early physical needs.

For males, overprotective parenting and parenting stress related to their child having genitoplasty prior to, but not later than, 12 months of age. While some males exhibited clinically significant overprotection, the group overall did not. Perhaps male caregivers are more comfortable with waiting for genitoplasty because they are not taking primary responsibility for bathing, dressing, and changing the child. The mean age of genitoplasty in the later surgery group was 22.8 months (Table 2). Thus, it appears that some form of adaptation occurs between the first and second years of a child's life that results in greater comfort of males to proceed with genitoplasty. Perhaps this allows for more time to obtain education or the opportunity to obtain a second opinion.

Furthermore, it is unclear how sex differences in caregiver reactions to genitoplasty might contribute to family conflict surrounding the treatment plan for a child's DSD. While the majority of our participants reported intact marriages, we did not assess the quality of these relationships. Elevated parenting stress and overprotective could translate into positive or negative psychosocial development for children. Future studies should consider children's psychosocial development as it relates to their parents reactions to DSD.

\section{Limitations}

Several limitations to the current study qualify our results. First, the study was cross-sectional. As a result, we were unable to examine the trajectory of the outcome variables over time. Second, shared method variance is a potential issue because in many cases 2 caregivers of a single child completed the study. Although separate analyses were 
TABLE 4: Bivariate correlations to determine covariates for male and female guardians whose children underwent genitoplasty.

\begin{tabular}{|c|c|c|c|c|c|c|c|c|c|c|c|}
\hline Male Caregiver & 1 & 2 & 3 & 4 & 5 & 6 & 7 & 8 & 9 & 10 & 11 \\
\hline (1) Guardian Age & & .37 & .29 & -.05 & c & .37 & -.41 & -.38 & -.17 & -.24 & $-.49^{*}$ \\
\hline (2) Child Age & & & .33 & .36 & c & $1.00^{* *}$ & .35 & .28 & $-.90^{* *}$ & -.35 & -.10 \\
\hline (3) Annual Income & & & & .00 & c & .32 & .12 & .16 & -.12 & .06 & -.04 \\
\hline (4)Number of Children in Home & & & & & c & .36 & -.17 & -.12 & $-.48^{*}$ & -.28 & -.17 \\
\hline (5) Number of Caregivers in Home & & & & & & c & c & c & $\mathrm{C}$ & c & c \\
\hline (6) Illness Duration & & & & & & & .34 & .28 & $-.90^{* *}$ & -.36 & -.11 \\
\hline (7) BAI Total & & & & & & & & $.91^{* *}$ & -.35 & .05 & $.58^{*}$ \\
\hline (8) BDI-2 Total & & & & & & & & & -.20 & .14 & $.57^{*}$ \\
\hline (9) PPS Total & & & & & & & & & & $.57^{*}$ & .05 \\
\hline (10) CVS Total & & & & & & & & & & & .38 \\
\hline \multicolumn{12}{|l|}{ (11) PSI/SF Total } \\
\hline Female Caregiver & 1 & 2 & 3 & 4 & 5 & 6 & 7 & 8 & 9 & 10 & 11 \\
\hline (1) Guardian Age & & $.39^{*}$ & .25 & -.03 & -.12 & $.42^{*}$ & .05 & .07 & -.33 & .20 & .01 \\
\hline (2) Child Age & & & -.14 & .28 & $-.47^{*}$ & $.83^{* *}$ & -.05 & .07 & $-.56^{*}$ & .04 & .20 \\
\hline (3) Annual Income & & & & .00 & $.50^{* *}$ & -.35 & -.09 & -.12 & .09 & .05 & .00 \\
\hline (4) Number of Children in home & & & & & -.19 & .15 & -.01 & -.11 & -.35 & -.24 & -.16 \\
\hline (5) Number of Caregivers in home & & & & & & $-.46^{*}$ & .00 & -.25 & .18 & -.22 & -.02 \\
\hline (6) Illness Duration & & & & & & & .07 & .11 & $-.71^{* *}$ & .05 & .24 \\
\hline (7) BAI Total & & & & & & & & $.60^{* *}$ & .09 & .26 & $.50^{* *}$ \\
\hline (8) BDI-2 Total & & & & & & & & & .10 & .29 & $.64^{* *}$ \\
\hline (9) PPS Total & & & & & & & & & & .00 & -.03 \\
\hline (10) CVS Total & & & & & & & & & & & .13 \\
\hline (11) PSI/SF Total & & & & & & & & & & & \\
\hline
\end{tabular}

${ }^{*} P<.05 .{ }^{* *} P<.01 . \mathrm{c}=$ constant due to all male caregivers reporting 2 children in the household. Significant demographic and illness correlations with dependent variables of interest were included as covariates in applicable analyses. Numerals with variable name correspond to numeral on horizontal heading.

TABLE 5: Comparison of Genitoplasty.

\begin{tabular}{|c|c|c|c|c|c|c|c|}
\hline \multirow{2}{*}{ Male Caregiver } & \multicolumn{2}{|c|}{ Gentioplasy not Conducted } & \multicolumn{2}{|c|}{ Genitoplasy Conducted } & \multicolumn{3}{|c|}{ ANOVA/ANCOVA } \\
\hline & $M$ & $S E$ & $M$ & $S E$ & $F$ & $P$ & Partial $\eta^{2}$ \\
\hline BAI & 3.25 & 1.64 & 4.00 & 1.09 & 0.15 & .706 & 0.01 \\
\hline BDI-2 & 5.00 & 2.05 & 5.39 & 1.37 & 0.03 & .876 & 0.00 \\
\hline PPS & 31.53 & 2.07 & 32.88 & 1.04 & 0.28 & .602 & 0.02 \\
\hline CVS & 6.27 & 1.02 & 4.33 & 0.64 & 2.31 & .143 & 0.10 \\
\hline $\mathrm{PSI} / \mathrm{SF}$ & 67.75 & 6.04 & 59.56 & 4.03 & 1.27 & .270 & 0.05 \\
\hline Female Caregiver & $M$ & $S E$ & $M$ & $S E$ & $F$ & $P$ & Partial $\eta^{2}$ \\
\hline BAI & 6.77 & 1.73 & 7.14 & 1.16 & 0.03 & .860 & 0.00 \\
\hline BDI-2 & 14.00 & 2.57 & 11.35 & 1.72 & 0.74 & .396 & 0.02 \\
\hline PPS & 30.83 & 2.16 & 32.89 & 1.20 & 0.57 & .455 & 0.02 \\
\hline CVS & 5.75 & 1.26 & 6.59 & 0.81 & 0.31 & .581 & 0.01 \\
\hline $\mathrm{PSI} / \mathrm{SF}$ & 82.23 & 5.41 & 67.55 & 3.62 & 5.08 & .030 & 0.11 \\
\hline
\end{tabular}

Male caregiver: genitoplasty not conducted $(n=8)$, genitoplasty conducted $(n=18)$. Female caregiver: genitoplasty not conducted ( $n=13)$, genitoplasty conducted $(n=29)$.

conducted for female and male caregivers in an attempt to address this issue, it is possible that shared method variance impacted the results. Additionally, caregivers in the current study were predominantly Caucasian, married, and reported a relatively high annual income. As such, the current results may not generalize to other populations. Some of the participants are caregivers of younger or older children at different developmental stages than what the outcome measures were developed to assess. Finally, the type and extent of education and counseling surrounding their child's DSD and treatment plan were unavailable for caregiver participants. We were therefore unable to consider these potentially important factors when analyzing the data. 
TABLE 6: Comparison of Time of Genitoplasty.

\begin{tabular}{|c|c|c|c|c|c|c|c|}
\hline \multirow{2}{*}{ Male Caregiver } & \multicolumn{2}{|c|}{ Genitoplasy $\leq 12$ months } & \multicolumn{2}{|c|}{ Gentioplasy $>12$ months } & \multicolumn{3}{|c|}{ ANOVA/ANCOVA } \\
\hline & $M$ & $S E$ & $M$ & $S E$ & $F$ & $P$ & Partial $\eta^{2}$ \\
\hline BAI & 4.46 & 1.50 & 2.80 & 2.42 & 0.34 & .568 & 0.02 \\
\hline BDI-2 & 6.23 & 1.69 & 3.20 & 2.73 & 0.89 & .359 & 0.05 \\
\hline PPS & 31.45 & 0.83 & 27.43 & 1.36 & 6.16 & .028 & 0.32 \\
\hline CVS & 4.39 & 0.63 & 3.20 & 1.01 & 0.99 & .334 & 0.06 \\
\hline $\mathrm{PSI} / \mathrm{SF}$ & 64.95 & 3.69 & 45.53 & 6.22 & 6.70 & .021 & 0.31 \\
\hline Female Caregiver & $M$ & $S E$ & $M$ & SE & $F$ & $P$ & Partial $\eta^{2}$ \\
\hline BAI & 7.72 & 1.49 & 6.18 & 1.90 & 0.41 & .529 & 0.02 \\
\hline BDI-2 & 10.33 & 2.11 & 13.00 & 2.69 & 0.61 & .442 & 0.02 \\
\hline PPS & 30.84 & 1.36 & 31.16 & 1.69 & 0.02 & .883 & 0.00 \\
\hline CVS & 7.17 & 1.00 & 5.64 & 1.28 & 0.89 & .354 & 0.03 \\
\hline PSI/SF & 68.39 & 4.14 & 66.18 & 5.30 & 0.11 & .745 & 0.00 \\
\hline
\end{tabular}

Male caregiver: genitoplasty under 12 months $(n=13)$, genitoplasty over 12 months $(n=5)$. Female caregiver: genitoplasty under 12 months $(n=18)$, genitoplasty over 12 months $(n=11)$.

\section{Conclusion}

In response to the increasing awareness of cosmetic and functional limitations of reconstructive surgery in the context of genital ambiguity [4], some medical centers have adopted a more conservative approach in the surgical treatment of children with DSD [26]. Our data indicate that choosing not to proceed with genitoplasty at all, as well as doing so very early in life, is associated with higher levels of stress and maladaptive parenting characteristics for some caregivers. Perhaps caregivers are fearful that others will observe their child's ambiguous genitalia and question their gender. Other fears may include the belief that atypical genital anatomy leads to atypical sexual behavior. Additionally, caregivers who opt out of genitoplasty may do so because they lack the financial resources necessary to obtain surgical treatment for their child. Decisions around whether or not to have genitoplasty for a child born with ambiguous genitalia are complex and difficult for parents to make. Future qualitative studies are needed in this group to better understand why some caregivers respond negatively when choosing to not have genitoplasty for their child.

Finally, though we found an association between early surgical events and parental stress and overprotection among male caregivers, we cannot address cause and effect. It is possible that stressed, overprotective men were more eager to have earlier surgery for their child. Alternatively, caregivers who choose genitoplasty within the first 12 months of their child's life may feel that they were rushed into the decision or incompletely informed. As caregivers' negative responses to DSD can impact psychological development of children, future research should examine how stress and parenting characteristics surrounding genitoplasty decisions made within the first year or life might translate into later adjustment problems for children.

Indeed, our data suggest that while some parents may be coping poorly with surgical decisions for their child, others are resilient. For the time being, it is necessary to assure that parents receive education about the pros and cons of genitoplasty for their child, including adequate time to process this information. Part of this education should include acknowledgement of the possible risks to not having surgery or having surgery within the first year of life. Additionally, because surgery is irreversible, counseling to help relieve parental stress surrounding genitoplasty decisions should be offered. More work is needed in this understudied area to optimize outcome for children born with ambiguous genitalia.

\section{Additional Note}

Although some of the items from the CVS, PSI-SF, and PPS may not be appropriate for children of every developmental stage, we elected to retain all original items rather than make arbitrary decisions a priori as to which items were not developmentally appropriate. Additionally, each of these measures has been used in previous research for parents of children of all ages $[6,8-10,19]$.

\section{Acknowledgments}

This research was supported by the Study Network of Pediatric Endocrinologists (SNoPE) of the Lawson Wilkins Pediatric Endocrine Society, the Presbyterian Health Foundation (PHF) of Oklahoma, and Sigma Theta Tau International Honor Society of Nursing. The authors wish to thank the caregivers who participated in this study and Drs. Brad Kropp and Dominic Frimberger for their helpful comments.

\section{References}

[1] P. A. Lee, C. P. Houk, S. F. Ahmed, et al., "Consensus statement on management of intersex disorders," Pediatrics, vol. 118, no. 2, pp. e488-e500, 2006.

[2] M. Hines, S. F. Ahmed, and I. A. Hughes, "Psychological outcomes and gender-related development in complete androgen insensitivity syndrome," Archives of Sexual Behavior, vol. 32, no. 2, pp. 93-101, 2003. 
[3] K. Schützmann, L. Brinkmann, M. Schacht, and H. RichterAppelt, "Psychological distress, self-harming behavior, and suicidal tendencies in adults with disorders of sex development," Archives of Sexual Behavior, vol. 38, no. 1, pp. 16-33, 2009.

[4] V. Schönbucher, K. Schweizer, L. Rustige, et al., "Sexual quality of life of individuals with 46,XY disorders of sex development," Journal of Sexual Medicine. In press.

[5] T. Mazur, "Disorders of sex development: psychological issues and outcomes," in Lawson Wilkins Pediatric Endocrine Society Annual Meeting, Gender Medicine: Spanning the Specialties, Honolulu, Hawaii, USA, 2008.

[6] A. E. Kazak, L. Barakat, K. Meeske, et al., "Posttraumatic stress, family functioning, and social support in survivors of childhood leukemia and their mothers and fathers," Journal of Consulting and Clinical Psychology, vol. 65, no. 1, pp. 120-129, 1997.

[7] L. L. Mullins, J. M. Chaney, V. L. Hartman, et al., "Child and maternal adaptation to cystic fibrosis and insulin-dependent diabetes mellitus: differential patterns across disease states," Journal of Pediatric Psychology, vol. 20, no. 2, pp. 173-186, 1995.

[8] L. L. Mullins, C. Wolfe-Christensen, A. L. Hoff Pai, et al., "The relationship of parental overprotection, perceived child vulnerability, and parenting stress to uncertainty in youth with chronic illness," Journal of Pediatric Psychology, vol. 32, no. 8, pp. 973-982, 2007.

[9] K. K. Anthony, K. M. Gil, and L. E. Schanberg, "Brief report: parental perceptions of child vulnerability in children with chronic illness," Journal of Pediatric Psychology, vol. 28, no. 3, pp. 185-190, 2003.

[10] C. J. M. Colletti, C. Wolfe-Christensen, M. Y. Carpentier, et al., "The relationship of parental overprotection, perceived vulnerability, and parenting stress to behavioral, emotional, and social adjustment in children with cancer," Pediatric Blood and Cancer, vol. 51, no. 2, pp. 269-274, 2008.

[11] G. N. Holmbeck, S. Z. Johnson, K. E. Wills, et al., "Observed and perceived parental overprotection in relation to psychosocial adjustment in preadolescents with a physical disability: the mediational role of behavioral autonomy," Journal of Consulting and Clinical Psychology, vol. 70, no. 1, pp. 96-110, 2002.

[12] C. Wiesemann, S. Ude-Koeller, G. H. G. Sinnecker, and U. Thyen, "Ethical principles and recommendations for the medical management of differences of sex development (DSD)/intersex in children and adolescents," European Journal of Pediatrics, vol. 169, no. 6, pp. 671-679, 2009.

[13] J. E. Dayner, P. A. Lee, and C. P. Houk, "Medical treatment of intersex: parental perspectives," Journal of Urology, vol. 172, no. 4, pp. 1762-1765, 2004.

[14] J. M. Crawford, G. Warne, S. Grover, B. R. Southwell, and J. M. Hutson, "Results from a pediatric surgical centre justify early intervention in disorders of sex development," Journal of Pediatric Surgery, vol. 44, no. 2, pp. 413-416, 2009.

[15] P. Kline, The Handbook of Psychological Testing, Routledge, London, UK, 2nd edition, 1999.

[16] A. T. Beck, N. Epstein, G. Brown, and R. A. Steer, "An inventory for measuring clinical anxiety: psychometric properties," Journal of Consulting and Clinical Psychology, vol. 56, no. 6, pp. 893-897, 1988.

[17] K. A. Wilson, E. de Beurs, C. A. Palmer, et al., "Beck anxiety inventory," in The Use of Psychological Testing for Treatment Planning and Outcomes Assessment, M. E. Maruish, Ed., pp. 971-992, Lawrence, Erlbaum, Mahwah, NJ, USA, 2nd edition, 1999.
[18] A. T. Beck, R. A. Steer, and G. K. Brown, Manual for the Beck Depression Inventory-II, Psychological Corporation, San Antonio, Tex, USA, 1996.

[19] D. J. A. Dozois, K. S. Dobson, and J. L. Ahnberg, "A psychometric evaluation of the Beck Depression Inventory-II," Psychological Assessment, vol. 10, no. 2, pp. 83-89, 1998.

[20] M. Thomasgard, W. P. Metz, C. Edelbrock, and J. P. Shonkoff, "Parent-child relationship disorders. Part I. Parental overprotection and the development of the Parent Protection Scale," Journal of Developmental and Behavioral Pediatrics, vol. 16, no. 4, pp. 244-250, 1995.

[21] B. W. C. Forsyth, S. M. Horwitz, J. M. Leventhal, J. Burger, and P. J. Leaf, "The child vulnerability scale: an instrument to measure parental perceptions of child vulnerability," Journal of Pediatric Psychology, vol. 21, no. 1, pp. 89-101, 1996.

[22] R. R. Abdin, Parenting Stress Index/short Form Manual, Western Psychological Services, Los Angeles, Calif, USA, 1990.

[23] D. K. Carson and R. W. Schauer, "Mothers of children with asthma: perceptions of parenting stress and the mother-child relationship," Psychological Reports, vol. 71, no. 3, pp. 1139$1148,1992$.

[24] T. Wysocki, K. Huxtable, T. R. Linscheid, and W. Wayne, "Adjustment to diabetes mellitus in preschoolers and their mothers," Diabetes Care, vol. 12, no. 8, pp. 524-529, 1989.

[25] L. Murray, F. Hentges, J. Hill, et al., "The effect of cleft lip and palate, and the timing of lip repair on motherinfant interactions and infant development," Journal of Child Psychology and Psychiatry and Allied Disciplines, vol. 49, no. 2, pp. 115-123, 2008.

[26] V. Pasterski, P. Prentice, and I. Hughes, "Consequences of the Chicago consensus on disorders of sex development (DSD): current practices in Europe," Archives of Disease in Childhood. In press. 